\title{
Analysis of Peer Review System Based on Fewness Distribution Function
}

\author{
Jian Zhou ${ }^{1, a}$, Ning Cai ${ }^{1, b^{*}}$ and Yanjun $\mathrm{Li}^{2, \mathrm{c}}$ \\ ${ }^{1}$ College of Electrical Engineering, Northwest University for Nationalities, China \\ ${ }^{2}$ school of Economics, Northwest University for Nationalities, China \\ azhoujianfrank@126.com, bcaining91@tsinghua.org.cn, c371222246@qq.com
}

${ }^{*}$ The corresponding author

Keywords: Peer review system; Skewness distribution; Probability model; Monte Carlo

\begin{abstract}
For dealing with randomness characteristic of the paper submitted and accepted, a probability arithmetic of reliability analysis for peer review system is presented on basis of skewed normal distribution and in combination with Monte Carlo simulation algorithm. The Approximate Probability and Mathematical Expectation indices can be obtained by Matlab simulation and M-C method. Based on the randomness analysis of the paper submitted and accepted principles. It is verified that this probability arithmetic is feasible. It is easier to figure out that we can have a more clear and objectives acknowledge for peer review system.
\end{abstract}

\section{Introduction}

Peer review [1] also called peer expert evaluation, it is a primary method of the qualitative evaluation [2], the peer experts who refers to the same subject or field or the same research direction. At present, one of the main standard [2-3] is depend on whether there is peer review when foreign academia or scholar to estimate the quality of the academic journals. It's make no mistake that such reviews and how to implement peer review is crucial to the quality monitoring of the academic journals, thus the international periodical usually held on peer-reviewed conference on a regular basis. With the wide recognition and popularization of the peer review system [4]. And thus puts forward more strict requirements for the authors and the reviewers. In most cases, however, peer review process were occured before the paper has published. The database of the ISI [5] has lay much emphasis upon whether with or without peer review system when they select publications. In the majority review system of the database of the SCI. SSCI. Skopos. A\&HCI, this task is accomplished by initial review, final review three stages [6]. The reviewers should in line with the academic level of the manuscript to provide reasons for the decision with clarity and objectivity, and according to the shortages of the initials, the reviewers put forward some reference proposals on how to further improve the article. On the basis of upon, ultimate liability by the editors to decide whether the manuscript can be accepted. Thus it can be seen that, the peer review process is a greatly important link in paper quality assessment.

\section{Peer Review System}

The peer review system has already became a hot topic in the industry for the past few years. Whether a journal will accept or submit an article must depends on the peer review principles. If one of the reviewers chose to refuse the article [7], this means that the article will be submitted. In other words, the article will be accepted on condition that all of the reviewers chose accept. This is what we often call—one ticket is overruled make [7-8].

The Form of the Peer Review. The mainly form [8] of the current peer-reviewed as follows:

Single-Blind Review. The author's name is open to the reviewers, nevertheless, the reviewer's name by means of an anonymous type for the all authors. According to a research survey made by ALPSP indicated that about $60 \%$ of the journal adopt single blind review form [9].

Double-Blind Review. Namely, the reviewer's name and the author's name are not open to each 
other. Take a Double-Blind Review aimed at limiting the reviewers favorably on discrimination.

Public-Review. The reviewer's name and the author's name are open to each other. It is highly recognized for public review is a better way to prevent the irresponsible comments from some singular reviewers. But the most reviewers unwilling open their own name in public, and therefore it is difficult to promote public review form [10]. About $88 \%$ of the journal to the author feedback is withheld the identity of the reviewers' comments according to a study from ALPSP. The BMJ statistics show that $73 \%$ of the periodicals take the form of two referees in each paper, $24 \%$ of the periodicals take the form of three referees or more than three referees in each paper.

\section{The Shortage of the Peer Review}

(1) Subjectivity: If the editor unwilling to hire some articles, the manuscript reviewers were sent more harsh reviewers aim to reach the purpose of rejection.

(2) Tendency: The review process aspects of discrimination against the author's nationality, native language, gender, affiliation, etc. The tendency of the review process has become more apparent when the reviewers competes with the authors. Particularly, the authors who come from Non-English-Speaking countries and not well-known institutions.

(3) Abuse of power: The abuse power of the reviewers including that reviewers plagiarism the unpublished outcome of the review process, reviewers are deliberately delay published the articles of the authors who have potentially competing relationship with the reviewers.

In view of the shortage and existing problems of peer review, Willamson [11] was suggested that in order to improve the quality of the peer review by taking public review or in the form of Internet. Certainly, the peer review can prevent fabricate the results from experiments and tamper the data at some degree [11-12].

\section{Probability Model}

So far as our knowledge is concerned, according to a survey made by ALPSP the average accepted rate of the general journals are about $37 \%$. Certainly the data of the core journals are lower than average 4\%-8\%. In general speaking, the most of the journals will random select 2 to 4 individuals from reviewers alternative database as the final referees. Therefore, an article can be collected must depend on the all referees. However this article want to point out that the employment rate of a journal is not the same as the average employment rate of the referees. The paper presented a model of the probability measure and Mathematical Expectation analysis [12] according to the peer review principle.

It is shown by statistical results (Table 1) that with the increase of the author recommended reviewers in proportion, the employment rate increased significantly.

Table 1 The Accepted rate of the authors recommendation and editors selection

\begin{tabular}{|l|c|c|}
\hline Sources & Totals & Accepted Rate \\
\hline Authors Recommendation & 255 & $45.67 \%$ \\
\hline Editors Selection & 500 & $54.32 \%$ \\
\hline
\end{tabular}


Table 2 The Accepted rate of different disciplines

\begin{tabular}{|c|c|c|}
\hline Subject & Total Contributions & Accepted Rate \\
\hline Life Science & 190 & $41.1 \%$ \\
\hline Geo Science & 180 & $36.7 \%$ \\
\hline Information & 131 & $31.3 \%$ \\
\hline Engineering & 99 & $40.4 \%$ \\
\hline Physics & 72 & $31.9 \%$ \\
\hline Chemistry & 20 & $40.1 \%$ \\
\hline
\end{tabular}

Model Description. For the peer review system composed of reviewers, by the one ticket is overruled make definition. It is concluded that the probability model as follows:

Probability Distribution Function:

$$
f(x)=\frac{1}{\omega} \frac{2}{\pi} \cdot e^{-\frac{(x-\xi)^{2}}{2 \omega^{2}}} \cdot \int_{-\infty}^{\infty} e^{-\frac{t^{2}}{2}} d t, x \in(-\infty,+\infty)
$$

Mathematical Expectation:

$$
E(x)=\xi+\omega \delta \cdot \frac{2}{\pi}, \text { where } \delta=\frac{\alpha}{1+\alpha^{2}}
$$

Maximum likelihood estimates for and can be computed numerically, If a closed-form expression is needed, theme thod of moments can be applied to estimate from the skew, by inverting the skewness equation. This yields the estimate as follows:

$$
|\delta|=\frac{\pi}{2} \cdot \frac{\left|r_{1}\right|^{\frac{2}{3}}}{\left|r_{1}\right|^{\frac{2}{3}}+[(4-\pi) / 2]} \text { where } \delta=\frac{\alpha}{1+\alpha^{2}}
$$

Consequently, the Variance is as follows:

$$
D(x)=\omega^{2}\left(1-\frac{2 \delta^{2}}{\pi}\right)
$$

\section{Conclusion}

By the above analysis of the probability, apparently, if a journal collection rate is specified approximation, then we can according to the reviewer records from different reviewers to match the most ideal reviewers from reviewers alternative storehouse. In fact, there are a lot of uncertainties during the period of review, such as reference source, authors recommendation, source of subject project, etc. To sum up, $p\left(\sum\right)$ and $p(\Pi)$ may not be the best index to quantitatively measure the final employment rate of the journals. In this sense, a better way to evaluate the extent of journals should be to measure how to find out the best candidate from the journal's database. With the following simulation, one can directly figure out which patterns is made for journals. 


\section{References}

[1] Garcia-Perez M., "A multidimensional extension to Hirschs h-index”, Scientometrics, vol. 81, no. 3, pp. 779-785, 2009.

[2] Jones T., Huggett S., Kamalski J., "Finding a way through the scientific literature: Indexes and measures", World Neurosurgery, vol. 76, p. 36, 2011.

[3] Zhang C.-T., "A proposal for calculating weighted citations based on author rank", EMBO Reports, vol. 10, no. 5, pp. 416-417, 2009.

[4] Bornmann L., Daniel H. D., "What do citation counts measure? A review of studies on citing behavior", Journal of Documentation, vol. 64, no. 1, pp. 45-80, 2008.

[5] Bouabid, H.Evaluation of Moroccan research using a bibliometric based approach: investigation of the validity of the h -index [J]. Scientometrics, 2009, 78 (2); 203-217.

[6] Jian C. G.Xia G.Exploring the h-index at patent level [J]. Journal of the American Society for Information Science and Technology, 2009, 60 (1): 35-40.

[7] Banks, M. G.An extension of the Hirsch index: Indexing scientific topics and compounds [J]. Scientometrics, 2006, 69 (1): 161-168.

[8] Jin B H. H-index: An evaluation indicator proposed by scientist. Science Focus (in Chinese), 2006, 1(1): 8-9.

[9] Van Raan A F J. Comparison of the Hirsch-index with standard bibliometric indicators and with peer judgment for 147 chemistry research groups. Scientometrics, 2006, 67(3): 491-502.

[10] Hirsch J E. An index to quantify an individual's scientific research output. Proc Natl Acad Sci USA, 2005, 102(46): 16569-16572.

[11] Braun T, Glänzel W, Schubert A. A Hirsch-type index for journals. The Scientist, 2005, 19(22): $17-18$.

[12] Glänzel W. On the h-index-A mathematical approach to a new measure of publication activity and citation impact. Scientometrics, 2006, 67(2): 315-321. 